\title{
Petra Radtke
}

\section{Die Frau, die mit dem Wolf tanzt}

Jahrelang wurde ich von einem Facharzt zum anderen geschickt. Mein Körper spielt mit mir Katz und Maus - er stellt schubweise wichtige Organfunktionen ein und zwingt mich dadurch zwischenzeitlich sogar in den Rollstuhl. Ich bin ein menschliches Stehaufmännchen geworden und begreife, dass die „Götter in Weiß" auch nur Menschen sind und ratlos meinem Zustand gegenüberstehen. Pillen und Spritzen missbilligt mein Körper und reagiert mit allergischen Schocks. Die meisten Ärzte werfen das Handtuch, wenn ich als Patient nicht in die Schublade passe, die das Krankheitsbild ihnen scheinbar vorgibt. Oft werde ich wie eine Außerirdische bestaunt - nur um zu guter Letzt in die Psychoschiene geschoben zu werden.

Von einem jungen Arzt musste ich mir sogar anhören, dass ich mein Bein, das gebeugt in einer Lähmung erstarrt war, nur nicht bewegen will! „Ich werde mich jetzt auf ihr Bein legen, und Sie werden sehen - es lässt sich bewegen!“, sagte er zu mir. Gesagt, getan ... jedoch ohne Erfolg - was ihn aber nicht nachdenklich machte, sondern nur missmutig stimmte. Er empfahl mir, bei einem Orthopäden vorstellig zu werden - diesen hatte ich aber ohne Erfolg schon zuvor konsultiert.

Im Internet fand ich einen so genannten ,Ganzheitsarzt' und machte einen Termin. Ich dachte, dieser Arzt sieht endlich mal meinen Körper als Ganzes und kann mir deshalb sicher gut helfen. Mich empfing ein Mann in weißem Gewand und Jesuslatschen. Er sah aus wie ein selbst ernannter Guru. Ich musste ihm von meiner Familie erzählen, die Großeltern eingeschlossen. In dem Arztzimmer standen bis zur Decke hoch Regale mit Arzneifläschchen, nach Buchstaben sortiert. Er fragte nicht nach Vorbefunden, ging nur kurz auf meine Beschwerden ein. Ich musste mich auf eine Liege setzen und er erzählte mir, dass mein Großvater Schuld an meiner Krankheit wäre. Denn er musste schon als junger Mann als Soldat in zwei Weltkriege ziehen und habe nun seine Schmerzen auf mich übertragen. Nun wurde der Arzt immer mehr zum Guru. Denn ich hatte einen mich sehr liebenden Opa, der mich immer vor allem Bösen dieser Welt bewahrt hat. Eigentlich hätte ich jetzt die Konsultation abbrechen können, aber ich war gespannt darauf, was sich der Guru noch einfallen ließ. Dieser schrieb auf einen Zettel einen Spruch, den ich jetzt jeden Abend vor dem Schlafengehen laut aufsagen sollte. Inhaltlich war es eine Bitte an meinen Opa, den Fluch von mir zu nehmen. Weiter ging es damit, dass er mehrere Arzneifläschchen aus dem riesengroßen Regal nahm und sie abwechselnd auf meinen Bauch stellte. Dabei murmelte er immer einen Spruch und stellte dann das Fläschchen entweder wieder zurück oder schüttete ein paar Kügelchen daraus in eine Papiertüte. Diese

Ә OpenAccess. ( 2021 Petra Radtke, publiziert von De Gruyter. (cc)BY-NC-SA Dieses Werk ist lizenziert unter einer Creative Commons Namensnennung - Nicht kommerziell - Weitergabe unter gleichen Bedingungen 4.0 International Lizenz. https://doi.org/10.1515/9783110713336-037 
Tütchen überreichte er mir lächelnd mit dem Hinweis, täglich diese Mittel zu nehmen. Beim Abschied erinnerte er mich noch daran, an der Anmeldung die Rechnung für die Konsultation nicht zu vergessen und einen neuen Termin $\mathrm{zu}$ machen. Ich ging um eine Erfahrung reicher.

Schließlich, nach einer monatelangen Arztodyssee, kam eine Ärztin auf die Idee, mein Blut auf bestimmte Antikörper zu untersuchen. Dann kam der Augenblick der Wahrheit. Die junge Ärztin schaute auf das vor ihr liegende Ergebnis meines Blutbildes und wurde sichtlich nervös. „Ich muss den Professor dazu holen!“, meinte sie, verschwand und kam mit dem Professor zurück. Seine Worte erreichen mich kaum. Ich verstehe nur: „Autoimmunkrankheit - systemischer Lupus, sehr selten, unheilbar - Gefahr von Organversagen und Tumorerkrankungen ...“ Vollkommen benommen von dieser Diagnose fuhr ich nach Hause. Immer wieder hallten die Worte des Arztes in meinem Kopf wider: „Sie werden nicht mehr arbeiten können!“ Mein ganzes Leben stand am Abgrund. Meinen Traumberuf als Pädagogin werde ich nie mehr ausüben können. Wie soll ich das aushalten? Außerdem bedeutet die Krankheit für mich, ab sofort ein Schattendasein führen zu müssen. Denn UV-Licht stärkt das Immunsystem und damit den Lupus, den Wolf in mir. Hinzu kommt: Ich bin alleinerziehende Mutter.

Meine beiden Kinder sind die Liebe meines Lebens. Wie soll ich ihnen beibringen, dass im Sommer Ausflüge an den Badesee ab sofort tabu für mich sind? Wie soll es finanziell weitergehen? Denn eine Erwerbsminderungsrente $\mathrm{zu}$ beantragen, dauert. Schon seit einigen Jahren lebe ich von Sozialhilfe, da ich wegen der Krankheit nicht arbeitsfähig bin und mein früherer Ehemann keinen Unterhalt zahlt. Außerdem muss ich einen ambulanten Rheumatologen finden, der sich mit Lupus auskennt. Aber ich werde nicht aufgeben. Ich habe in meinem Leben schon oft bewiesen, dass ich ein Stehaufmännchen bin.

Ich lebe nun schon zehn Jahre mit meinem täglichen Begleiter, dem Wolf. Es war nicht einfach, vieles im Leben musste ich ändern oder mich davon für immer verabschieden. Aber ich habe meine Kinder, die mir Mut geben, täglich weiterzumachen. Durch den Wolf haben sich einige Menschen, von denen ich dachte, dass sie zu meinen Freunden zählen, für immer verabschiedet. Aber ich habe einen neuen Freundeskreis gefunden. Durch Selbsthilfegruppen fand ich Ärzte, die mir mit Rat und Tat zur Seite stehen. Sie helfen mir, auch wenn es nicht einfach ist, meine Erkrankung medikamentös einzustellen, denn zu dem Wolf haben sich noch andere seltene Erkrankungen hinzugesellt.

Ich führe Kalender über die guten Tage im Monat: Diese bekommen eine lachende Sonne. Wenn mir ihr Lächeln mindestens zehnmal auf einem Kalenderblatt entgegenleuchtet, war es ein guter Monat. Denn das bedeutet, dass ich an zehn Tagen hinaus in die Natur konnte. Mit dem Wolf werde ich mich zwar nie anfreunden können. Doch durch die Erkrankung habe ich gelernt, die kleinen 
Freuden des Lebens $\mathrm{zu}$ entdecken und $\mathrm{zu}$ genießen. Ich bin eine begeisterte Hobbyfotografin und besonders die Makrofotografie hat es mir angetan. Damit habe ich einen neuen Blick auf die kleine Welt da draußen bekommen. Heuschrecken sehen dann auf meinen Fotos aus wie mächtige Saurier, die besonders meinen Sohn in seiner Kindheit so beeindruckten. In Wassertropfen spiegelt sich eine ganze Welt. Abends bin ich oft mit dem Rad unterwegs und beobachte Vögel, die mir ein wunderschönes Konzert spielen. Meine Fotos werden häufig in Zeitungen und Zeitschriften veröffentlicht und es gab auch schon eine Ausstellung. An schlechten Tagen, an denen der Wolf zubeißt und ich auf meinem Sofa bleiben muss, entschwebe ich mithilfe meiner treuen Freunde, den Büchern, in eine andere Welt. Ich lerne in den Geschichten neue Freunde kennen, erlebe Abenteuer auf eine besondere Art, reise zu Orten auf der Welt, die ich leider in diesem Leben nicht mehr besuchen kann. Über meine Leseabenteuer schreibe ich auf meinem Bücherblog und auf Facebook. Überhaupt habe ich durch das Internet, das für mich das Tor zur Welt ist, viele neue Freunde gefunden. Wir tauschen uns aus und machen uns Mut - so wird das Leben mit dem inneren Wolf erträglicher. Auch meine Hypnosetherapie hat mir viel gebracht: Den Ängsten, die mit der Erkrankung auftauchten, konnte ich gestärkt entgegentreten. Selbst der Umgang mit den täglichen Schmerzen wurde durch die Hypnose besser und depressive Phasen kann ich nun schneller überwinden.

Ich möchte mit meiner Geschichte Mut machen, nie im Leben aufzugeben. Man darf sich nie scheuen, Hilfe zu suchen - auch in einer fast ausweglos scheinenden Situation. Bei Ärzten muss man sein Selbstwertgefühl wahren und bei seltenen Erkrankungen helfen Selbsthilfegruppen mit ihren Erfahrungen. Wenn eine Planung im Leben durch schwere Erkrankungen nicht mehr möglich ist, setzt man sich eben täglich kleine Ziele und belohnt sich dann für deren Erfüllung. Ein Hobby kann einem sehr viele Glücksgefühle und Erfüllung geben. Für mich sind meine Kinder und meine Eltern ein starkes Bindeglied zum Leben. Auch mein Freundeskreis hilft mir mit Rat und Tat. Es lohnt sich, jeden Tag weiterzuleben - denn das Leben ist so schön - trotz alledem!

Meine Wünsche an eine gute medizinische Versorgung bei seltenen chronischen Erkrankungen:

- Diagnostik: Die Beschwerden des Patienten ernst nehmen. Wenn Überweisung zum Facharzt notwendig, Schreiben des überweisenden Arztes, welche Diagnostik schon stattfand und welcher Verdacht besteht. Hilfe bei Terminsuche beim Facharzt oder in Radiologie. Bessere Zusammenarbeit der Ärzte untereinander. Es kann einfach nicht sein, dass es wie bei mir fast zehn Jahre dauert, bis eine Ärztin auf die Idee kam, Antikörper im Blut auf systemischen Lupus zu testen, damit ich nun endlich die richtige Diagnose hatte und behandelt werden konnte. 
- Wissen über seltene Erkrankungen: Die Ärzte müssen besser geschult werden, damit sie die Beschwerden der Patienten nicht als psychologisches Problem abtun, weil ihnen Kenntnisse über Diagnostik oder Beschwerden bei seltenen Erkrankungen fehlen. Für Ärzte müsste es ein Infotelefon geben, wo sie von Experten Hinweise zur Behandlung von Patienten bekommen. Oft reagieren Patienten hier auf Medikamente allergisch oder atypisch. Ich habe dann oft von Ärzten gehört, dass sie mit mir am Ende der Fahnenstange sind und mir nicht mehr helfen können. Meine Erfahrungen sind, dass ich mit Bauchbeschwerden sogar von Fachärzten abgewiesen wurde, da sie sahen, dass auf der Überweisung vom Rheumatologen stand, dass ich an systemischem Lupus erkrankt war. So bekam ich keinen Termin und wurde zum Rheumatologen zurückgeschickt. Dieser wies mich in eine Klinik ein, in der verschiedene Untersuchungen - etwa eine Darmspiegelung - gemacht wurden. Doch die Ursache meiner Beschwerden blieb ein Rätsel. Mit der Diagnose Reizdarm wurde ich in die Psychoschiene gesteckt und unbehandelt nach Hause entlassen. Erst ein Professor aus einer anderen Stadt, den ich in meiner Not um Rat fragte, las sich die Ergebnisse meiner Diagnostik durch und gab mir telefonisch den Rat, meine Gewebeproben mit Verdacht auf systemische Mastozytose nach München zu senden. Vier Wochen später hatte ich meine gesicherte Diagnose. Doch der Weg dorthin dauerte vier Jahre. Dabei wäre es eine einfache Blutbestimmung gewesen, die den Verdacht aufgrund meiner starken Beschwerden in Richtung Mastozytose gelenkt hätte.

- Ausnahmeregelung für Budget der Ärzte: Für Ärzte, die chronisch Kranke mit seltenen Erkrankungen behandeln, muss es Ausnahmeregelungen beim Budget geben. $\mathrm{Zu}$ oft musste ich den Arzt wechseln, da mir notwendige Medikamente verwehrt wurden: Dadurch wäre das Ärztebudget zu sehr belastet worden.

- Bessere Prüfung von Medikamenten: Es kann nicht sein, dass Nofallmedikamente mit Stoffen belastet sind, die ihrerseits allergische Reaktionen hervorrufen können. Das betrifft übrigens auch Kontrastmittel, Narkosemittel oder Mittel, die man zur Darmreinigung vor der Darmspiegelung trinken muss. Ich muss seit Jahren für ein Magenmittel, ohne das ich nichts mehr essen könnte, über 50 Euro selbst bezahlen. Das Mittel, das die Kasse bezahlt, vertrage ich nicht wegen der Zusatzstoffe. Auch für Mittel gegen Magenund Darmbeschwerden oder Vitaminmangel durch Mangelernährung müsste es Sonderregelungen geben, denn die Beschwerden werden hervorgerufen durch allergische Reaktionen auf notwendige Lebensmittel.

- Reha-Einrichtungen: Es müssen mehr Informationen über seltenen Erkrankungen an Reha-Einrichtungen gegeben werden und es sollte die Möglichkeit einer längeren Rehazeit (jetzt drei Wochen) eingerichtet werden. Da unsere 
Körper sehr stark auf Behandlungen reagieren, brauchen wir zwischen den Behandlungen längere Ruhezeiten. Informationen über Reha-Einrichtungen, die seltene Krankheiten aufnehmen, müssen besser an Patienten und Krankenkassen gegeben werden.

- Behindertenstatus: Die Mitarbeiter der Sozialämter, die für die Einstufung der Grade der Behinderung verantwortlich sind, müssen besser geschult werden. Oftmals werden die Ausweise in viel zu niedriger Gradstufe ausgestellt, da man zu wenig über die jeweilige Krankheit weiß. Für die seltenen Krankheiten müssen Listen erstellt werden, nach denen die Mitarbeiter leichter eine Einstufung vornehmen können. Zum Beispiel bei meiner Erkrankung systemische Mastozytose würde ich mir wünschen, ohne Probleme die Bestätigung einer Begleitperson zu bekommen, da ich auf viele Dinge allergisch reagiere. Zwar führe ich immer ein Notfallset mit mir, aber wenn ich nicht mehr reagieren kann, wissen fremde Menschen meist nicht, wie sie mir helfen können.

- Einstufung Behindertengrad G: Mit diesem Grad der Behinderung hat man die Möglichkeit, eine Wertmarke zu kaufen, die freie Fahrt mit öffentlichen Verkehrsmitteln deutschlandweit ermöglicht. Leider gilt das nicht für schnelle Zugverbindungen wie den ICE. Aber für mich ist es körperlich einfach nicht möglich, z.B. drei Stunden von Leipzig nach Berlin mit zweimal umsteigen zu fahren, um meine Tochter zu besuchen. Ich würde es sehr begrüßen, wenn diese schnelle Zugverbindungen in die Verordnung zum freien Fahren für Behinderte mit dem Buchstaben $\mathrm{G}$ mit aufgenommen würde, denn ich weiß, dass es vielen Betroffenen ähnlich geht.

- Medizinische Versorgung im Notfall: Im Akutfall bin ich oft nicht in der Lage, mit öffentlichen Verkehrsmitteln zum Arzt zu fahren. Doch ich habe kein Auto und für ein Taxi reicht meine Rente meist nicht aus. Es gibt dann keine Möglichkeit, ärztlichen Rat einzuholen oder zu notwendigen radiologischen Untersuchungen zu kommen. Vor einigen Jahren wurden Fahrten zu ambulanten Untersuchungen noch von der Krankenkasse bezahlt. Auch hier sollte noch einmal geprüft werden, ob für uns Patienten eine Ausnahmeregelung möglich wäre.

- Osteopathie: Für an Lupus- und Mastozytose erkrankte Patienten gibt es nur wenige physische Behandlungen, die unser Körper verträgt. Eine davon ist die Osteopathie, die aber nun leider nicht mehr von der Krankenkasse übernommen wird. Ich habe selbst erlebt, wie ich an Unterarmstützen mit starken Schmerzen in der LWS zu einer damals noch von der Kasse bezahlten Behandlung ging. Mit wenigen Handgriffen erkannte der Therapeut, dass es nicht die LWS, sondern die Nieren waren, die durch Verklebungen diese Schmerzen hervorriefen. Dabei hatte ich noch Glück, denn nur ein paar Tage später wäre es ohne die osteopathische Behandlung wohl zu einem Nieren- 
versagen gekommen. Nach einer einstündigen Behandlung konnte ich ohne Schmerzen wieder laufen und das hielt auch mehrere Monate an. Ich würde mich freuen, wenn die Kosten dieser Art der Behandlung wieder von der Kasse übernommen werden, denn es würde mir viele Schmerzen ersparen und meine Bewegungsfähigkeit deutlich verbessern.

Zusammenfassend möchte ich sagen, dass man als chronisch Kranker mit einer seltenen Erkrankung in unserem Gesundheitssystem immer wieder auf Grenzen stößt, die beim genaueren Hinsehen gar nicht notwendig wären. Auch chronisch kranke Menschen möchten am gesellschaftlichen Leben teilhaben und sich nicht durch ihre Krankheit ihr Leben bestimmen lassen.

\section{Ergänzung zur Reha für chronisch Kranke}

Als chronisch kranke Person ist es ist immer besonders schwer, eine Reha-Einrichtung zu finden, da für eine erfolgreiche Reha-Behandlung auf die Besonderheiten der chronischen Krankheit eingegangen werden muss. Bei mir betrifft es den systemischen Lupus und die systemische Mastozytose.

Im Jahr 2019 hatte ich zwei Unfälle mit mehreren Knochenbrüchen. Dadurch war es für mich notwendig, mehrere Monate im Rollstuhl zu sitzen. Nachdem ich wieder laufen konnte, beantragte mein Arzt mit mir zusammen eine Reha mit dem Ziel, die Beweglichkeit zu verbessern. Da durch die systemische Mastozytose starke Lebensmittelallergien bestehen, schrieb der Arzt es auch in den Antrag und ging in einem Arztbrief auf die Besonderheiten, die meine chronischen Krankheiten bedeuten, ein.

Ich suchte im Internet entsprechende Kliniken aus und rief dort an, um die Möglichkeiten einer Reha in der jeweiligen Einrichtung zu klären. Ich erhielt eine Zusage und meine Unterlagen wurden im Januar 2020 an diese Klinik gesendet. Im März 2020 hätte ich anreisen können, doch Corona machte einen Strich durch die Rechnung: Die Klinik auf Usedom wurde für mehrere Monate geschlossen.

Ende Mai erhielt ich die schriftliche Zusage, für Ende August meine Reha beginnen zu können. Ich freute mich sehr darauf, die Koffer waren gepackt und der Fahrdienst durch die Kasse war organisiert. Zwei Tage vor der Anreise kam plötzlich ein Anruf von der Klinik, dass meine Reha nicht stattfinden könnte, der Grund: meine Lebensmittelallergien. Für mich war das völlig unverständlich, da dieses Problem schon ein halbes Jahr vorher besprochen wurde, schließlich war es schriftlich im Antrag und Arztbrief enthalten und in der Patientenbroschüre des Heimes wird besonders die eigene Küche mit Absprachen bei der Zubereitung der Speisen für Patienten mit Lebensmittelallergien hervorgehoben. 
Auf meine Nachfrage kam dann die Antwort der Klinik telefonisch, dass ich anreisen könnte, wenn ich mich selbst verpflege. Das würde bedeuten, in der Stadt selbst Lebensmittel einzukaufen, mittags würden für mich nur gekochte Kartoffeln zubereitet werden können. Für mich als gehbehinderte Person aber ist dies eine Unmöglichkeit. Auch die Krankenkasse, die auch nur telefonisch von der Absage unterrichtet wurde, legte mir nah, auf diesen Vorschlag nicht einzugehen.

Durch die ganze Aufregung bekam ich einen Nervenzusammenbruch und einen schweren Schub, der mich tagelang ans Bett fesselte. Ich finde es unmöglich, wenn man so mit chronisch schwerkranken Menschen umgeht. Wir sind schon durch unsere Krankheiten vom normalen gesellschaftlichen Leben ausgeschlossen und eine Rehamaßnahme soll doch gerade dabei helfen, unsere Selbstständigkeit solange wie möglich zu erhalten und den Gesundheitszustand zu verbessern.

Die Krankenkasse versprach mir, die bestätigte Reha könne auch noch später stattfinden. Ich telefonierte wieder mit einigen Einrichtungen, besprach die Probleme und bekam in einer anderen Rehaklinik einen Platz für 2021. Doch die Freude darüber hielt nicht lange, denn eine Woche später kam die Nachricht, dass auch diese Klinik mich abgelehnt hatte, als sie meine Kurunterlagen erhalten haben.

Ich rief in der Kurklinik an und bekam die Auskunft, dass keine Patienten mit Lupuserkrankung in diesem Heim behandelt werden. Nachdem ich betonte, dass ich nicht wegen meiner Lupuserkrankung, die gut eingestellt ist, zur Reha fahren soll, sondern um die Unfallfolgen zu beseitigen, folgte ein nochmaliges Gespräch mit dem dortigen Arzt. Dann kam der erlösende Anruf: Eine überglückliche Mitarbeiterin teilte mir mit, dass meine Reha im März/April 2021 stattfinden könne. Ich hoffe bloß, dass es wirklich so sein wird und nicht wieder eine kurzfristige Absage erfolgt.

Ich weiß, dass Lupuspatienten nicht gern gesehen werden, denn bei uns müssen einige Abstriche in der therapeutischen Behandlung gemacht werden. Einige Behandlungen, z.B. Strombehandlungen, Moorpackungen usw., dürfen nicht durchgeführt werden. Außerdem müssen längere Pausen zur Erholung zwischen den Maßnahmen erfolgen, um keinen Schub zu provozieren.

Ich kenne auch andere Mitglieder unserer Selbsthilfegruppe, die voller Vorfreude zu einer Reha über hunderte Kilometer gefahren sind und dort nach zwei Tagen aus den vorhergenannten Gründen wieder nach Hause geschickt wurden. Auch mir wurde vor einigen Jahren zur Begrüßung durch die Ärztin gesagt, dass wenn ich einen Lupusschub bekäme, ich sofort nach Hause geschickt werde. Auch eine Verlängerung, die die Kasse auf jeden Fall genehmigt hätte, wurde abgelehnt. Das ist in meinen Augen eine Diskriminierung von chronisch Kranken. 
Durch bessere Aufklärung über seltene Krankheitsbilder könnten solche Probleme sicher beseitigt werden. Für uns Kranke, die wir auf regelmäßige Rehamaßnahmen angewiesen sind, wäre es ein großer Schritt zur Verbesserung unseres Krankheitsverlaufs und zur Vermeidung von Pflegefällen. 